\title{
Intravascular large B-cell lymphoma: an elusive diagnosis with challenging management
}

\author{
Akash Mukherjee, MD, ${ }^{a}$ and Ibrahim F Ibrahim, $\mathrm{MD}^{\mathrm{b}}$ \\ ${ }^{a}$ Department of Stem Cell Transplantation and Cellular Therapy, MD Anderson Cancer Center, Houston, Texas, ${ }^{b}$ Department of \\ Internal Medicine, Division of Hematology and Oncology, University of Texas Southwestern, Dallas, Texas
}

$\mathrm{I}$ ntravascular large B-cell lymphoma (IVBCL) is an aggressive and systemically disseminated disease that affects the elderly, with a median age of diagnosis around 70 years and no gender predilection. It is a rare subtype of extranodal diffuse large B-cell lymphoma (DLBCL) characterized by selective growth of neoplastic cells within blood vessel lumen without any obvious extravascular tumor mass. Hence, an absence of marked lymphadenopathy and heterogeneous clinical presentation make it difficult to diagnose accurately and timely, with roughly half of the cases found postmortem in previous case reports. ${ }^{1,2}$ The exact incidence of this disease is not known, but more recently, the accuracy of diagnosis of this type of lymphoma has improved with random skin and bone marrow biopsy. ${ }^{1,2}$ We present here a clinical case of this disease with an atypical presentation followed by a detailed review of its clinical aspects.

\section{Case presentation and summary}

A 43-year-old white woman with a history of hypothyroidism and recurrent ovarian cysts presented to clinic with 3 months of loss of appetite, abdominal distension, pelvic pain, and progressive lower-extremity swelling. A physical examination was notable for marked abdominal distension, diffuse lower abdominal tenderness, and pitting lowerextremity edema. No skin rash or any other cutaneous abnormality was noted on exam. Laboratory test results revealed a lactate dehydrogenase $(\mathrm{LDH})$ level of $1652 \mathrm{U} / \mathrm{L}$ and a CA-125 level of $50 \mathrm{U} / \mathrm{mL}$ (reference range, 0-35 U/mL). No significant betahuman chorionic gonadotropin and alpha-fetoprotein levels were detected. Computed-tomographic (CT) imaging revealed small bilateral pleural effusions and gallbladder wall thickening with abdomi- nal wall edema, but it was otherwise unrevealing. An echocardiogram showed normal cardiac structure and function, with a left ventricular ejection fraction of $60 \%$. No protein was detected in the patient's urine, and thyroid function tests were unrevealing. Doppler ultrasound studies of her lower extremities and abdomen revealed no thrombosis. Given the patient's continued pelvic pain, history of ovarian cysts, and elevation in CA-125, she underwent a laparoscopic total abdominal hysterectomy and bilateral salpingoopherectomy.

Histologic examination revealed neoplastic cells involving only the vascular lumina of the cervix, endomyometrium, bilateral fallopian tubes, and bilateral ovaries (Figure 1). Immunohistochemistry stains were positive for CD5, CD20, PAX-5, CD45, BCL-2, and BCL-6 and focally positive for CD10. Peripheral smear showed pseudo-Pelger-Huet cells with 5\% atypical lymphoma cells (Figure 2). Complete staging with positron-emission and CT (PET-CT) imaging revealed no metabolic activity, and a bone marrow biopsy showed trilineage hematopoiesis with adequate maturation and less than $5 \%$ of the marrow involved with large B-cell lymphoma cells. A diagnosis of IVBCL was made.

Further work-up to rule out involvement of the central nervous system (CNS) included magneticresonance imaging (MRI) of the brain and cerebrospinal fluid (CSF) cytology and flow cytometry, which were negative.

Our patient underwent treatment with 6 cycles of infusional, dose-adjusted R-EPOCH (rituximab, etoposide phosphate, prednisone, vincristine sulfate, cyclophosphamide, doxorubicin hydrochloride) and 6 doses of prophylactic intrathecal chemotherapy with alternating methotrexate and cytarabine (Ara-C),

Accepted for publication November 2, 2018. Correspondence: Akash Mukheriee, MD; AMukherjee21@mdanderson. org. Disclosures: The authors report no disclosures or conflicts of interest. JCSO 2018;16(5):e280-e282. (C2018 Frontline Medical Communications. doi: https://doi.org/10.12788/jcso.0425 
and initial and subsequent CSF sampling showed no disease involvement. Consolidation with high-dose chemotherapy with R-BEAM (rituximab, carmustine, etoposide, Ara-C [cytarabine], melphalan) followed by rescue autologous stem cell transplantation (ASCT) was performed, and the patient has remained in clinical and hematologic remission for the past 24 months.

\section{Discussion}

\section{Clinical presentation}

The clinical manifestation of this disease is highly variable, and virtually any organ can be involved. Besides causing constitutional symptoms, including fatigue, B symptoms, and decline in performance status, heterogeneity of the clinical presentation depends on the organ system involved. One of the exceptional features of this disease is the difference in clinical presentation based on the geographical origin of the patient. ${ }^{2-4}$

Western-variant IVBCL has a higher frequency of CNS and skin involvement, whereas Asian-variant IVBCL shows preferential involvement of bone marrow with hemophagocytosis, hepatosplenomegaly, and thrombocytopenia. However, these 2 clinical variants have no difference in clinical outcome, except with the cutaneous-variant kind. ${ }^{24} \mathrm{~A}$ retrospective case series of 38 Western-variant IVBCL cases showed that $55 \%$ of patients had B symptoms with poor performance status. ${ }^{3}$ Brain and skin were the organs that were most frequently involved, with $68 \%$ of patients having involvement of at least 1 of those organs. Ten patients in this case series had disease that was exclusively limited to the skin and described as a "cutaneous variant" of IVBCL. ${ }^{3}$

Similarly, a retrospective case series of 96 cases of Asianvariant IVBCL showed B symptoms in $76 \%$ of patients, with predominant bone marrow involvement in $75 \%$ of patients, accompanied by hemophagocytosis in $66 \%$ and hepatosplenomegaly and anemia/thrombocytopenia in $77 \%$ and $84 \%$ of the patients, respectively. ${ }^{4}$ This difference in clinical presentation might have existed as a result of ethnic difference associated with production of inflammatory cytokines, including interferon gamma, tumor necrosis factor-alpha, interlukin-1 beta, and soluble interlukin-2 receptor, with levels of soluble interlukin-2 receptor found to be significantly higher in Asian patients than non-Asian patients. $^{2}$

\section{Diagnosis}

Involved organ biopsy is mandatory for establishing the diagnosis of IVBCL. Laboratory findings are nonspecific, with the most common abnormality being increased serum $\mathrm{LDH}$ and beta- 2 microglobulin levels observed in $80 \%$ to $90 \%$ or more of patients. Despite its intravascular growth pattern, IVBCL was associated with peripheral blood involvement in only $5 \%$ to $9 \%$ of patients. ${ }^{1}$

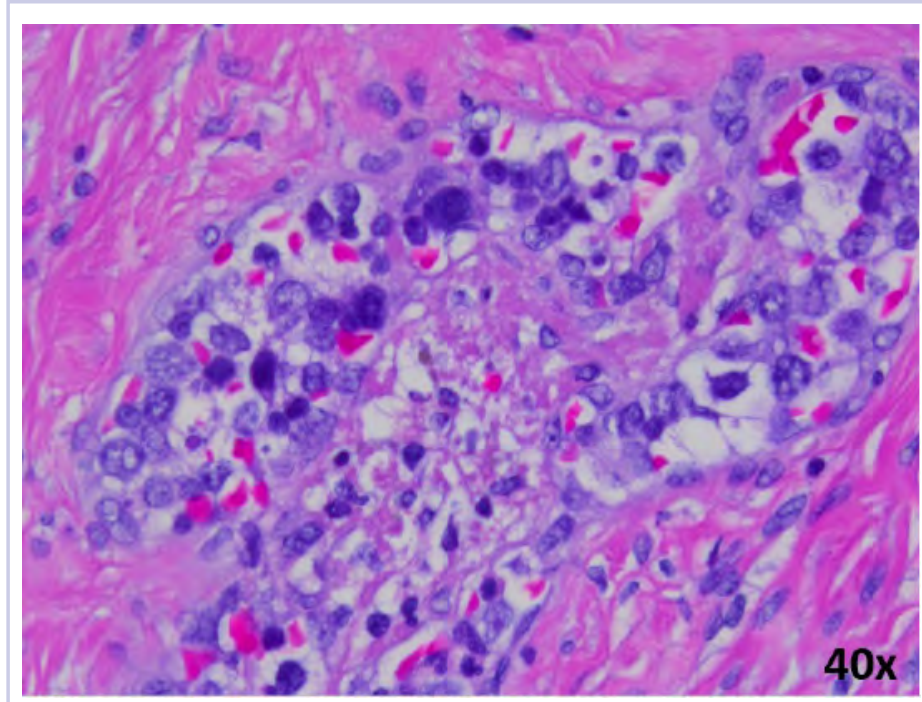

FIGURE 1 Large atypical lymphoma cells (some hyperchromatic/dark nuclei, many with vesicular/bubbly chromatin, can be seen with some red blood cells) within the vascular lumina (H\&E stain, 40x)

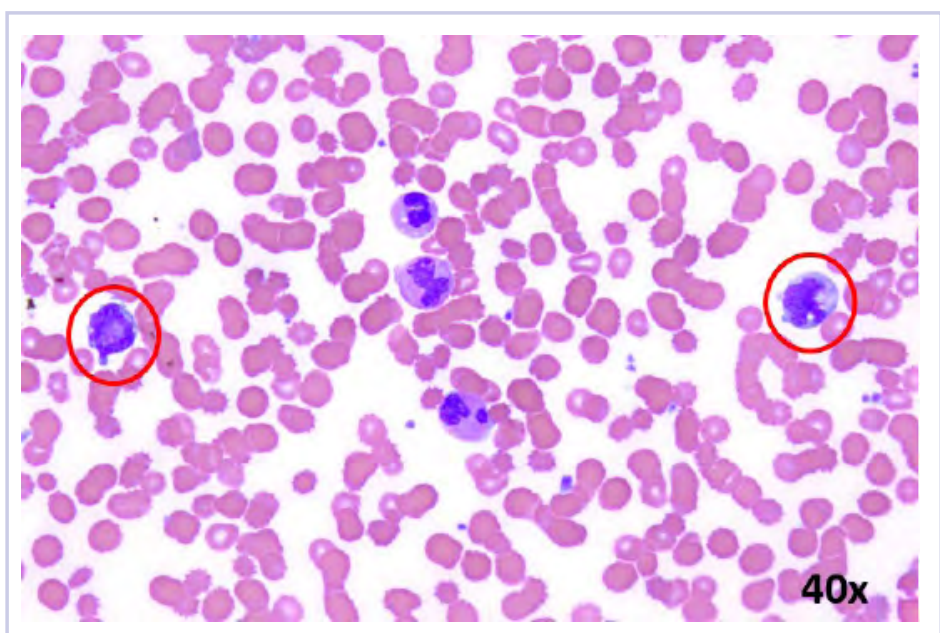

FIGURE 2 Peripheral blood smear showing large atypical lymphoid cells circled red (Wright-Giemsa Stain, 40x)

\section{Staging}

Clinical staging work-up suggested for IVBCL patients by International Extranodal lymphoma study group in 2005 included physical examination (with emphasis on nervous system and skin), routine blood studies, peripheral blood smear, total body CT scan with contrast or PET-CT scan, MRI brain with contrast, CSF cytology, and bone marrow or organ biopsy. ${ }^{1}$ The role of fluorodeoxyglucose-PET scan is controversial but can be helpful to detect unexpected locations for biopsy and to assess treatment response. ${ }^{5,6}$

\section{Morphology and immunophenotyping}

In general, IVBCL histopathology shows large neoplastic lymphoid cells with large nuclei along with one or more 
nucleoli and scant cytoplasm within blood vessel lumen. Immunophenotypically, IVBCL cells mostly express nongerminal B-cell-associated markers with CD79a (100\%), CD20 (96\%), MUM-IRF4 (95\%), CD5 (38\%), and CD10 (12\%) expressions. IVBCL cells have been demonstrated to lack cell surface protein CD29 and CD54 critical to transvascular migration. Similarly, aberrant expression of proteins such as CD11a and CXCR3 allows lymphoma cells to be attracted to endothelial cells, which might explain their intravascular confinement. ${ }^{7}$

\section{Genetics}

No pathognomic cytogenetic abnormalities have been reported in IVBCL to date, and the genetic features of this disease are not yet completely understood., ${ }^{2,7}$

\section{Management}

IVBCL is considered a stage IV disseminated disease with an International Prognostic Index score of high-intermediate to high in most cases. Half of the patients with IVBCL who were treated with anthracycline-based chemotherapy relapsed and died within 18 months of diagnosis. One third of the relapses involved the CNS, thereby highlighting the importance of prophylactic CNS-directed Intrathecal therapy in an induction treatment regimen..$^{2-4}$ Ferreri and colleagues reported in their case series response rates of about $60 \%$, with an overall survival (OS) of 3 years of $30 \%$ in patients who were treated with anthracyclinebased chemotherapy. A multivariate analysis of the entire series showed cutaneous variant of the disease to be an independent favorable prognostic factor for OS. ${ }^{3}$

In the Murase and colleagues case series, the authors reported $67 \%$ response rates and a median OS of 13 months with CHOP (cyclophosphamide, doxorubicin hydrochloride, vincristine sulfate, prednisone) or CHOPlike regimens. Multivariate analysis showed older age, thrombocytopenia, and absence of anthracycline-based

\section{References}

1. Ponzoni M, Ferreri AJ, Campo E, et al. Definition, diagnosis, and management of intravascular large B-cell lymphoma: proposals and perspectives from an international consensus meeting. J Clin Oncol. 2007;25(21):3168-3173.

2. Shimada K, Kinoshita T, Naoe T, Nakamura S. Presentation and management of intravascular large B-cell lymphoma. Lancet Oncol. 2009;10(9):895-902.

3. Ferreri AJ, Campo E, Seymour JF, et al. Intravascular lymphoma: clinical presentation, natural history, management and prognostic factors in a series of 38 cases, with special emphasis on the 'cutaneous variant'. Br J Haematol. 2004;127(2):173-183.

4. Murase T, Yamaguchi M, Suzuki R, et al. Intravascular large B-cell lymphoma (IVLBCL): a clinicopathologic study of 96 cases with special reference to the immunophenotypic heterogeneity of CD5. Blood. 2007;109(2):478-485.

5. Miura Y, Tsudo M. Fluorodeoxyglucose-PET/CT for diagno- chemotherapy to be an independent negative prognostic factor for OS. ${ }^{4}$ Another retrospective analysis by Shimada and colleagues of 106 patients with IVBCL showed improved outcome with the addition of rituximab to CHOP-based chemotherapy (R-CHOP). Complete response rate (CR), 2-year progression-free survival, and OS were significantly higher for patients in rituximabchemotherapy group than for those in the chemotherapyalone group (CR, $82 \%$ vs $51 \%$, respectively, $P=.001$; PFS, $56 \%$ vs $27 \%$; OS, $66 \%$ vs $46 \%, P=.001$ ), thereby establishing rituximab with $\mathrm{CHOP}-$ based therapy as induction therapy for IVBCL patients. ${ }^{8}$

The role of high-dose chemotherapy followed by ASCT could also be used as consolidation therapy to improve clinical outcomes as reported in 7 patients, showing durable remission after transplant in these 2 case series. ${ }^{3,4}$ Another retrospective analysis of 6 patients with IVBCL who were treated with 6 cycles of $\mathrm{R}-\mathrm{CHOP}$ as induction therapy and consolidated with ASCT reported all patients to be alive and in complete remission after a median follow-up of 56 months. ${ }^{9}$ Based on the retrospective case series data by Kato and colleagues and considering that more than $80 \%$ of the patients with IVBCL were in the high-risk International Prognostic Index group, ASCT in first remission might be a useful treatment option for durable remission; however, because the median age for the diagnosis of IVBCL is about 70 years, ASCT may not be a realistic option for all patients.

\section{Conclusions}

IVBCL is a rare, aggressive, and distinct type of DLBCL with complex constellations of symptoms requiring strong clinical suspicion to establish this challenging diagnosis. Rituximab with anthracycline-based therapy along with prophylactic CNS-directed therapy followed by consolidative ASCT may lead to long-term remission. More research is needed into the genetic features of this disease to better understand its pathogenesis and potential targets for treatment.

sis of intravascular large B-cell lymphoma. Mayo Clin Proc. 2010;85(8):e56-e57.

6. Shimada K, Kosugi H, Shimada S, et al. Evaluation of organ involvement in intravascular large B-cell lymphoma by $18 \mathrm{~F}$-fluorodeoxyglucose positron emission tomography. Int J Hematol. 2008;88(2):149-153.

7. Orwat DE, Batalis NI. Intravascular large B-cell lymphoma. Arch Pathol Lab Med. 2012;136(3):333-338.

8. Shimada K, Matsue K, Yamamoto K, et al. Retrospective analysis of intravascular large B-cell lymphoma treated with rituximab-containing chemotherapy as reported by the IVL study group in Japan. J Clin Oncol. 2008;26(19):3189-3195.

9. Kato K, Ohno Y, Kamimura T, et al. Long-term remission after high-dose chemotherapy followed by auto-SCT as consolidation for intravascular large B-cell lymphoma. Bone Marrow Transplant. 2014;49(12):1543-1544. 